\title{
Management of Acute Ascending Cholangitis with Septic Shock on Top of Altered Anatomy after Old Road Traffic Accident: A Case Report
}

\author{
Alsaati H, Omar I*, Sharif $O$ and Alhamid WA
}

Department of General Surgery, King Hamad University Hospital, Bahrain

\begin{abstract}
Introduction: Acute cholangitis is a clinical syndrome characterized by fever, jaundice, and abdominal pain that develops because of stasis and infection in the biliary tract. The most frequent causes of biliary obstruction are biliary calculi, benign biliary stricture, and malignancy.

Case presentation: Here we report a case presented with septic shock and acute ascending cholangitis on top of an altered anatomy in the form of disruption of the head and Wirsung duct of the pancreas, complete avulsion of the CBD at the ampulla of vater and deformed duodenal bulb after an old road traffic accident sustained thirty-five years back. Initial resuscitation, and antibiotic therapy with urgent decompression have been done. Definitive surgery has been performed in the form of dismantling of prior cholecystojejunostomy, exploration and resection of common bile duct with cholecystectomy and Roux-en-Y hepaticojejunostomy with adhesiolysis.

Conclusions: Definitive surgery remained the mainstay of treatment for extrahepatic biliary and pancreatic trauma to prevent future life-threatening complications. MDT approach is the optimal choice for dealing with critically ill patients. Utilization of the full capabilities of endoscopic and interventional radiology services deemed mandatory for urgent and timely management.
\end{abstract}

Keywords: Acute cholangitis; Syndrome; Radiograph; Duodenal bulb

\section{Introduction}

Acute cholangitis is a clinical syndrome characterized by fever, jaundice, and abdominal pain that develops because of stasis and infection in the biliary tract. It is also referred to as ascending cholangitis. Cholangitis was first described by Charcot as a serious and life-threatening illness; however, it is now recognized that the severity can range from mild to life-threatening [1]. The most frequent causes of biliary obstruction in patients with acute cholangitis without bile duct stents are biliary calculi $(28 \%$ to $70 \%)$, benign biliary stricture ( $5 \%$ to $28 \%$ ), and malignancy ( $10 \%$ to $57 \%$ ) [2]. Acute cholangitis can also occur following endoscopic retrograde cholangiopancreatography (ERCP) (0.5\% to $1.7 \%)$, particularly therapeutic ERCP following stent placement, or postoperatively due to bile duct injury, or a stricture of biliary-enteric anastomosis. Rarely, the distal common bile duct may be obstructed by food, stones, or debris in patients with a biliary-enteric anastomosis (Sump syndrome) [3-5]. Here we report a case presented with septic shock and acute ascending cholangitis on top of an altered anatomy after an old road traffic accident sustained thirty-five years back.

\section{Case Presentation}

A 63-year-old male, who has a known case of diabetes mellitus on oral hypoglycemic presented to ED with an epigastric pain of 2 days duration with a pain score of $9 / 10$. He complained of nausea, vomiting, anorexia and chills as well. He has no known allergy; however, he has a significant surgical history of blunt abdominal trauma sustained after road traffic accident (RTA) which resulted in severe injuries 35 years ago. Such injuries which were vague and not known in a detailed manner on presentation as the patient had been managed in another country at the time. At presentation he was in shock and feverish; BP: 80/40 -HR: $106-\mathrm{Tem}: 40.1^{\circ} \mathrm{C}$-RR: $20 / \mathrm{min}-\mathrm{SO}_{2:} 98 \%$ on $2 \mathrm{~L}$ via nasal cannula. On examination, clinical jaundice was noticed, in addition to a median laparotomy scar. His abdominal examination showed moderate tenderness in the epigastric region. On presentation, he had an open bowel. The patient was managed as per ACLS protocol with fluid resuscitation and noradrenaline $0.5 \mathrm{mcg} / \mathrm{kg} / \mathrm{min}$ started. RBS checked and revealed $17.6 \mathrm{mmol} / \mathrm{l}$, so insulin infusion started. Laboratory results were as follows; Na: 122-K: 4.09-Crea: 118-Urea: 8.61-WBC: 9.31-Neut 94\%-Hb: 13.1 PLT: 168-AST: 219-ALP: 394-ALT: 227-GGT: 557-total bilirubin: 86-Direct bilirubin: 80-Amylase: 19.09-Lipase: 56 -CRP: 143. H1N1 -ve. VBG; PH: 7.22, $\mathrm{PCO}_{2}: 46, \mathrm{HCO}_{3}: 18$, Lac: 8.01 . He had a sinus rhythm on ECG. Bedside ECHO was normal with preserved LVEF $55 \%$.

Chest radiograph showed accentuated lung markings with multiple short air-fluid levels at the right side of the abdomen. On the abdominal radiograph, multiple faint radio-opaque shadows were noticed at the right hypochondrium, query GB stones. The patient had been admitted to ICU and sepsis protocol was applied. Furthermore, antibiotics were administered (Meropenem-Ciprofloxacin-Vancomycin) in addition to fluconazole. Abdominal ultrasound was done (Figures $1 \mathrm{~A}$ and $1 \mathrm{~B}$ ) revealing dilated $\mathrm{CBD}$ of $9.5 \mathrm{~mm}$ diameter with single stone seen at its proximal part measuring $10 \times 7 \mathrm{~mm}$. Dilated IHBRs were seen at both hepatic lobes. The gallbladder was contracted over stones with a thickened edematous wall. Urgent decompression by ERCP was deemed necessary. unfortunately, cannulation failed even with precut needle knife.

In the view of persistent tachycardia and high inotropic support, the patient was intubated electively for PTC, where cholangiogram was done which revealed IHBRD with dilated CBD and filling defects seen within likely of calcular nature. No obvious communication of distal end of CBD with the duodenum but only localized fixed opacified area

*Corresponding author: Islam Omar, Department of General Surgery, King Hamad University Hospital, Bahrain, Tel: +97334631360; E-mail islamfawzyomar@hotmail.com

Received October 07, 2018; Accepted October 10, 2018; Published October 12,2018

Citation: Alsaati H, Omar I, Sharif O, Alhamid WA (2018) Management of Acute Ascending Cholangitis with Septic Shock on Top of Altered Anatomy after Old Road Traffic Accident: A Case Report. J Clin Case Rep 8: 1177. doi: 10.4172/21657920.10001177

Copyright: @ 2018 Alsaati $\mathrm{H}$, et al. This is an open-access article distributed under the terms of the Creative Commons Attribution License, which permits unrestricted use, distribution, and reproduction in any medium, provided the original author and source are credited. 
is noted. $8.5 \mathrm{~F}$ external biliary drainage catheter was advanced (Figures $2 \mathrm{~A}$ and $2 \mathrm{~B})$

One day later, Echocardiogram was done giving the picture of type II myocardial injury due to underlying sepsis, with impaired systolic function-EF 30\%, markedly hypokinetic mid-lateral and mid to basal infero-lateral segments with hypokinetic remaining segments. Grade II LV diastolic dysfunction. Trivial MR. Mild to moderate TR with estimated systolic PAP of $35 \mathrm{mmHg}$. Cardiac profile showed CK: $340-$ CKMB: 9.53-Troponin-I: 11.1. The patient's situation had become clearer with ascending cholangitis, obstructive jaundice, septic shock, AKI, type 2 myocardial injury and deranged coagulation. Laboratory results were; WBC: 24.32-CRP: 202-GGT: 249-ALP: 172-T. Bil: 72.1-D. Bil: 57.64-Urae: 13.7-Creat: 177.6-ALT: 97-AST: 101-INR: 2.02-APTT: 48.4- APTT ratio: 1.86-PT: 23.2. Blood culture grew Escherichia coli.

Drainage from PTC catheter was nil and because of the deteriorated critical situation of the patient, decision had been taken to upsize the PTC catheter for decompressing his biliary system via $12 \mathrm{~F}$ catheter (Figures $3 \mathrm{~A}$ and $3 \mathrm{~B}$ ). On the third day of admission, the newly inserted PTC catheter drained $110 \mathrm{cc}$ and abdominal ultrasound was done (Figures 4A-4D) which showed PTC drainage tube inserted. Significant improvement regarding the previously seen dilatation of IHBRs and CBD was seen. A newly seen mild amount of free fluid at right iliac fossa was newly seen.

One day later, PTC drained $190 \mathrm{cc}$ clear bile, however, LFT was noted trending up with T. Bil: 108.3-D. Bil: 80.67. WBC also jumped to 32.12 with $90 \%$ neutrophilia. RFT manifested a significant rise; urea measured 23.7 and Create was 224.73. Moreover, the patient had gaseous distension of the abdomen that was confirmed by abdominal radiograph and hypoactive bowel sound with last bowel motion on the day of admission. MDT meeting gathered which in turn agreed to do a US abdomen and compare it with the previous one. CT abdomen with contrast would have been required if any significant abnormal finding in the US or any deterioration of the patient's condition had ensued. Results of US (Figures 5A-5E) were; more improvement regarding the previously seen dilatation of IHBRs and CBD with a change in the location, but with the same amount of free fluid seen at left iliac fossa due to change patient position. The gallbladder became more distended and shows large stone inside measuring $10 \times 8 \mathrm{~mm}$ with a significant decrease in gallbladder wall thickness (from $12 \mathrm{~mm}$ to $6 \mathrm{~mm}$ ). A newly seen rim of free fluid at peri-hepatic recess was noticed.

Over the next 7 days, the patient's condition had been dramatically improved. He was extubated, hemodynamically stable without inotropic support, shifted to the ward, the urinary catheter was removed, started oral feeding and opened his bowel. Laboratory findings had correlated well with the ameliorated clinical condition where, Urea: 5.16-Creat: 53.08-WBC: 7.9-Nuet 73.8\%-CRP: 41.7-T. Bil: 59.6-D. Bil: 41.2-GGT: 281-ALP: 239. Cholangiogram was done at the time (Figures $6 \mathrm{~A}$ and $6 \mathrm{~B})$ after removal of the previously inserted external biliary drainage catheter over guidewire-which showed dilated tortuous cystic duct with opacification of the proximal CBD distal to the cystic duct starting from confluence site with two distal CBD large filling defects likely of CBD stones, opacification of gallbladder as well as the gallbladder fundus-jejunal anastomotic site with a detectable multiple gall bladder stones of variable size. Advancement of external-internal 8.5F biliary drainage catheter was done with its internal end within the jejunal loop.

Over another week in the ward the patient condition had remained stable and planning for definitive surgical management was commenced, an official report of the old trauma was obtained which documented the following injuries sustained on this RTA, huge retroperitoneal bile stained haematoma, disruption of the head and Wirsung duct of pancreas, complete avulsion of the CBD at the ampulla of Vater and two duodenal perforations posteriorly and medially. Such injuries were managed by closure of duodenal perforations, $\mathrm{T}$-tube splint of the CBD, cholecystojejunostomy and sump drain insertion near the head of the pancreas. Postoperative investigations after the acute clinical course of his old trauma revealed that; on HIDA scan, bile enter the GIT through the cholecystojejunostomy. On ERCP contrast fails to enter either CBD or pancreatic duct, double contrast study showed deformed duodenal bulb. At the time since the patient was not complaining of steatorrhea nor jaundice he was advised to continue follow up with a possible need to convert his cholecystojejunostomy to choledochojejunostomy after further work up.

Taken into account this complicated anatomy, work up for definitive management started with CT with oral and IV contrast (Figures 7A-7C) which confirmed the following: sizable subcapsular collection along the right lateral and cranial aspects of the liver, with a maximal thickness of $4 \mathrm{~cm}$. In addition, internal/external biliary drainage catheter is noted coursing through the collection, liver, gallbladder and extending to the jejunal loops. There is also air within the lumen of the gallbladder and small amount of fluid within the Morison's pouch. The pancreas is not visualized in keeping with near complete lipomatosis and sutures were noted along the wall of the hepatic flexure of the colon.

Despite the continuing clinical improvement, the discovery of perihepatic collection mandated prompt intervention where, a US guided $12 \mathrm{~F}$ drainage catheter was inserted (Figures $8 \mathrm{~A}$ and $8 \mathrm{~B}$ ) within the perihepatic collection yielding clear bile, which was removed after five days when the drainage was about nil. Echocardiography was repeated showing normal left ventricular cavity size with preserved global systolic function (EF 55\%, previously 35\%) with no regional wall motion abnormality. The patient was discharged home with the PTC drain, in a good condition with a plan for definitive surgery after maximal optimization of his general condition.

Ten days later the patient came to hepatobiliary surgery clinic for assessment, the abdomen was soft and lax with midline laparotomy scar and 2 previous drain scars. PTC drain was connected and functioning. Management Plan was explained to the patient and his family, clarifying the need to have a definitive procedure to avoid future cholangitis and sepsis. In addition, the complexity and possible complications of the operative procedure (cholecystectomy plus excision of the previous cholecystojejunostomy with reconstruction of hepaticojejunostomy) were emphasized with cons and pros $v s$. endoscopic interventions.

An MDT meeting with radiology, GI and HB surgery was arranged. Referral to cardiology for assessment of fitness for surgery, a dietitian for high protein low carbohydrates diet and endocrinology for blood sugar control were done. Eventually, the patient was sent for preoperative anaesthesia consultation and booked for surgery after full optimization of his general condition and preoperative cholangiogram (Figure 9), which excluded any stricture or abnormal dilatation of intrahepatic biliary radicles, hepatic ducts or proximal CBD and confirmed the obstruction of CBD and dilatation and tortuosity of the cystic duct. The contrast flew from the gallbladder to the jejunum via cholecystojejunostomy.

The patient was admitted electively for exploratory laparotomy with dismantling of prior cholecystojejunostomy, exploration and resection of the common bile duct with cholecystectomy and Rouxen-Y hepaticojejunostomy with adhesiolysis. 
Citation: Alsaati H, Omar I, Sharif O, Alhamid WA (2018) Management of Acute Ascending Cholangitis with Septic Shock on Top of Altered Anatomy after Old Road Traffic Accident: A Case Report. J Clin Case Rep 8: 1177. doi: 10.4172/2165-7920.10001177
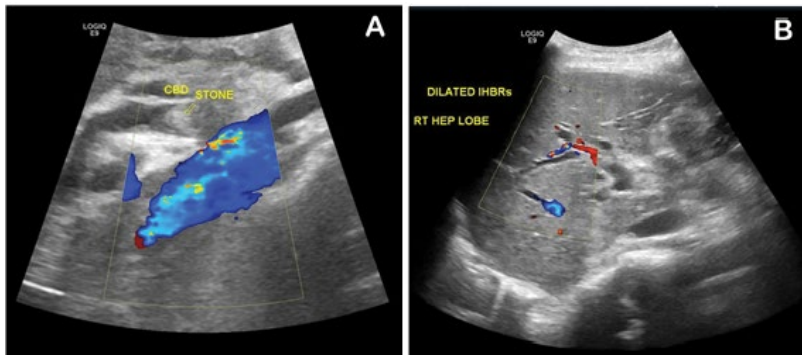

Figure 1: (A) CBD dilated with a stone within. (B) Dilated IHBR.
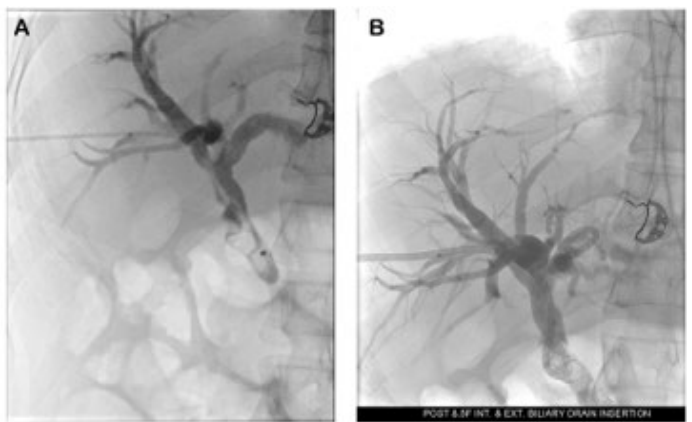

Figure 2: (A) Cholangiogram showing IHBRD, dilated $C B D$ and filling defects seen within, with no communication of distal end of CBD with the duodenum. (B) $8.5 \mathrm{~F}$ external biliary drainage catheter in place.
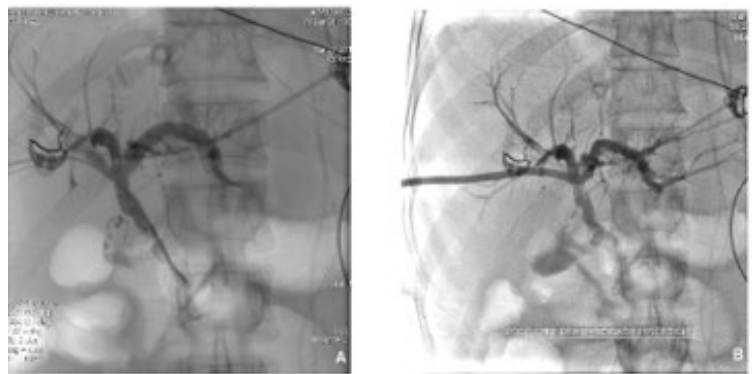

Figure 3: (A) Upsizing of the previously inserted 8.5. (B) F external biliary drainage was done by bigger one-12 $\mathrm{F}$.
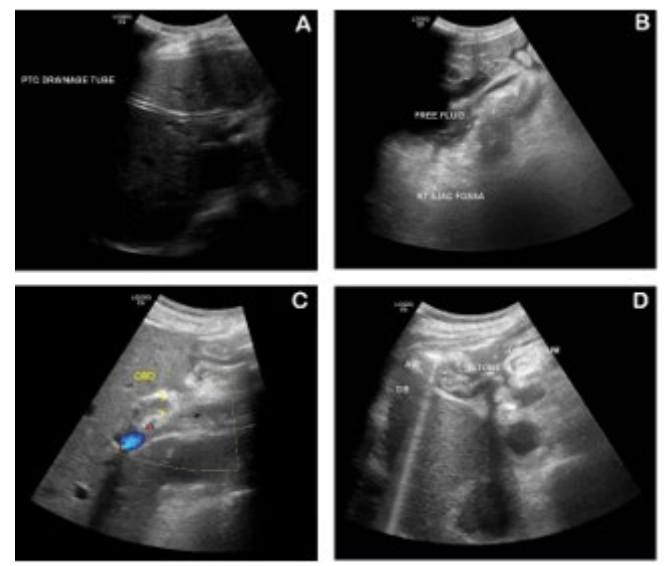

Figure 4: (A) PTC drainage tube inserted. (B) Free fluid at the right iliac fossa. (C) Significant improvement regarding the previously seen dilatation of IHBRs and CBD. (D) Contracted gallbladder with a thickened edematous wall with a stone within its lumen.
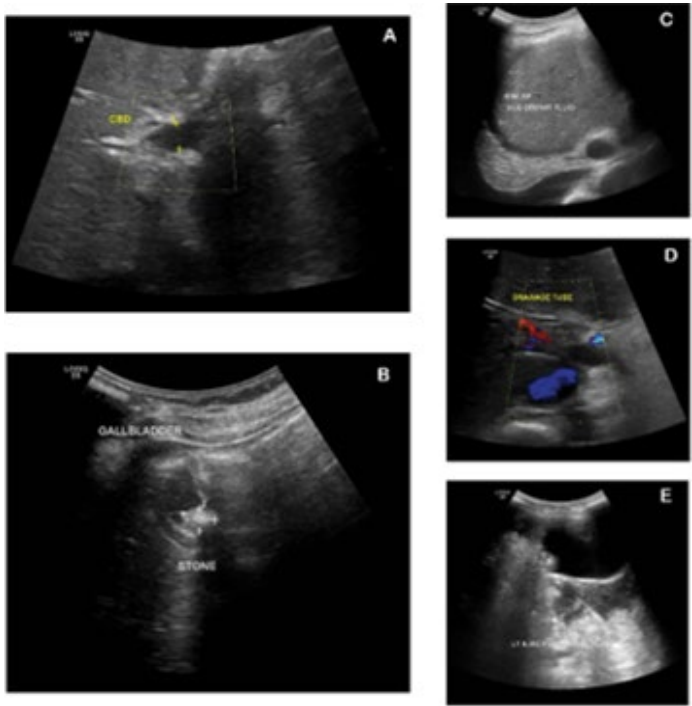

Figure 5: (A) Improvement of dilatation of IHBRs and CBD. (B) The gallbladder becomes more distended and shows large stone inside measures $10 \times 8 \mathrm{~mm}$. (C) Rim of free fluid at peri-hepatic recess. (D) Drainage tube in place. (E) Change in the location but with the same amount of free fluid seen now at left iliac fossa.
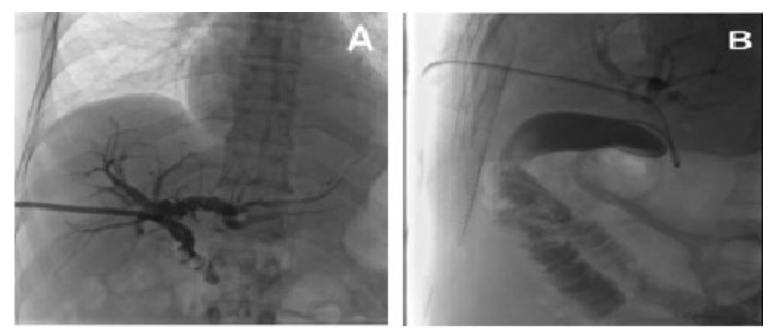

Figure 6: (A) Dilated tortuous cystic duct with opacification of the proximal CBD distal to the cystic duct starting from confluence site with two distal CBD large filling defects. (B) Opacification of gallbladder as well as the gallbladder fundus- jejunal anastomotic site.
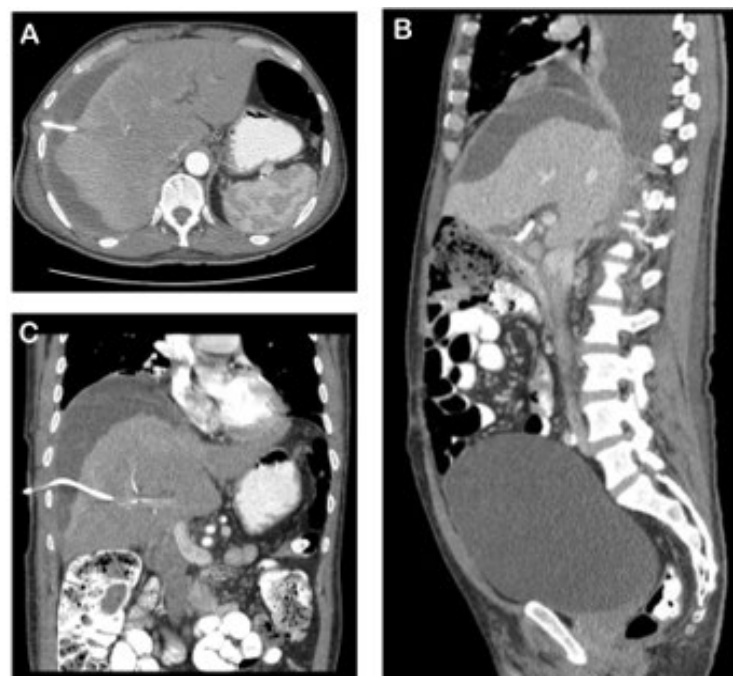

Figure 7: (A) Sizable subcapsular collection along the right lateral and cranial aspects of the liver. (B) Internal/external biliary drainage catheter is noted. (C) There is air within the lumen of the gallbladder with evidence of cholecystojejunostomy. 

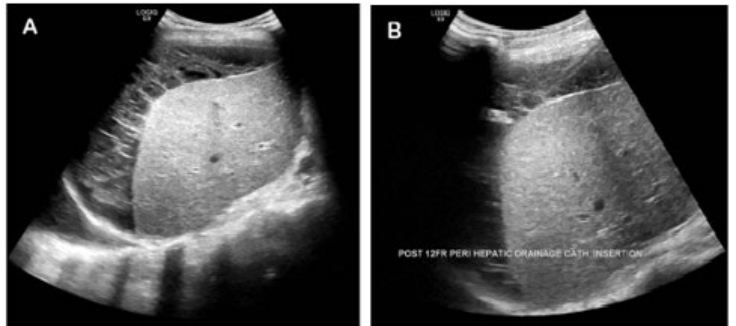

Figure 8: US-guided drainage of perihepatic collection (A) Before insertion. (B) After insertion of $12 \mathrm{~F}$ catheter

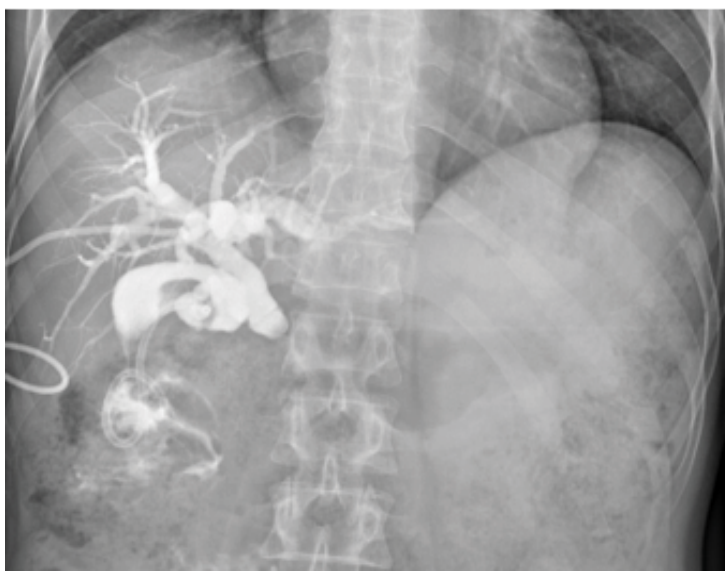

Figure 9: Cholangiogram showing, dilated tortuous cystic duct, obstructed distal CBD and patent cholecystojejunostomy.

\section{Discussion}

Obstruction of the biliary ducts and presence of a superposing bacterial infection are common features in cholangitis. Not every biliary obstruction is associated with cholangitis but there is surely a biliary obstruction in every cholangitis case [6-9]. Biliary obstruction is caused by choledocholithiasis mostly. Moreover, malignancy, benign strictures, and interventions to the biliary ducts may be the cause of biliary obstruction. Other mechanisms include extrinsic compression of the bile duct due to a duodenal periampullary diverticulum (Lemmel syndrome), inflammation secondary to acute pancreatitis, or an impacted stone in the cystic duct or neck of the gallbladder (Mirizzi syndrome). Other intrinsic causes of biliary obstruction include blood clots, and parasitic infections (mainly liver flukes and the roundworm Ascaris). Retained worm fragments can serve as a nidus for biliary stones and cause recurrent pyogenic cholangitis. Many other rare causes of cholangitis are reported in the literature, like recurrent cholangitis without stones with previous papillotomy, a picture suggestive of a causal relationship between heavy lifting and cholangitis, where increased intra-abdominal pressure can cause reflux into the common bile duct in the presence of a papillotomy [10]. A similar aetiology of recurrent cholangitis was described in patients who had undergone biliary bypass or pancreatoduodenectomy [11]. Another case report revealed another rare mechanism, where the condition was attributed to food impaction through a choledochoduodenal fistula leading to obstructive jaundice with ascending cholangitis progressing to sepsis [12]. External compression of the biliary tree from a ruptured renal angiomyolipoma (AML) of the right kidney and mucinous cystic neoplasm of the liver with extrahepatic growth have been reported as rare causes of ascending cholangitis $[13,14]$. A more unusual case of ascending cholangitis has been reported due to fractured ventriculogall bladder shunt fragments causing mechanical obstruction [15].

Common to all forms of biliary obstruction is elevated choledochal pressure which is deemed the inciting factor for the inflammatory process and sepsis. The biliary system works according to a pressure gradient where the pressure of the hepatic bile secretion is $120-150 \mathrm{~cm}$ $\mathrm{H}_{2} \mathrm{O}$ and the pressure in extrahepatic bile ducts is $100-150 \mathrm{~cm} \mathrm{H} \mathrm{H}_{2} \mathrm{O}$. So normally, bile secretion occurs according to these pressure values and bile fills into the gallbladder with a pressure of $12-18 \mathrm{~cm} \mathrm{H}_{2} \mathrm{O}$. Peristaltic contraction-relaxation of the sphincter of Oddi is the most important factor in the regulation of this pressure. Bile secretion from the liver is inhibited if the pressure exceeds $300 \mathrm{~cm} \mathrm{H}_{2} \mathrm{O}$. If the choledochal pressure exceeds $25 \mathrm{~cm} \mathrm{H}_{2} \mathrm{O}$, hepatic defense mechanisms against infection become useless, consequently, in $25-40 \%$ of the cases, the associated infection spreads into the intrahepatic canaliculi, and cholangio-venous reflux ensues, followed by the access to the hepatic veins and lymphatics, resulting in bacteriemia. Sepsis is common in the case of suppurative infections [16,17]. Isolated organisms are $E$. coli (27\%), Klebsiella (16\%), Enterococcus (15\%), Streptococcus (8\%), Enterobacter (7\%), and Pseudomonas aeruginosa (7\%) [18,19].

In our patient with altered anatomy multifactorial pathogenesis played a role to initiate such stormy clinical course. It is well known that risk factors for stone formation are bile duct dilatation, biliary stricture, and angulation of the common bile duct due to anatomical abnormalities [20,21]. The obstructed CBD, tortuous cystic duct and distorted duodenal bulb contributed to the formation of his CBD and gallbladder stones which in-turn led to the obstruction and creation of a vicious circle. Although bacterial colonization of the small intestine is not uncommon, it is rarely associated with widespread sepsis. Colonization itself is often a consequence of the failure of intestinal clearance of bacteria which may be due to dysmotility or altered anatomy. The prevalence of bacterial overgrowth varies in association with the predisposing medical disease or surgically modified anatomy, and whether patients studied are symptomatic [22-25]. Again, the altered anatomy with direct continuity of the patient biliary system with the jejunum through the previously formed cholecystojejunostomy provided a portal of entry through which E. coli gained access to his biliary system to induce his cholangitis and septic shock, where it was isolated from his blood culture.

Common bile duct (CBD) injuries from blunt abdominal trauma are rare. In fact, extrahepatic biliary tract injuries occur in 3\% to $5 \%$ of all abdominal trauma victims, with $85 \%$ resulting from penetrating wounds. Of the remaining $15 \%$, resulting from blunt trauma, the clear majority, $85 \%$, involve the gallbladder alone [26-28]. CBD injury occurs frequently at three areas of relative fixation of the biliary tract [29]:

1) The origin of the left hepatic duct,

2) The bifurcation of the hepatic ducts,

3) The pancreaticoduodenal junction.

Different mechanisms, even in combination, may produce rupture of the common bile duct: Compression of the ductal system against the vertebral column [30], sudden increase of intraluminal pressure in the gallbladder with a short and permeable cystic duct [31], and a "shearing force" producing avulsion of the common duct at its fixed part at the junction with the pancreas [32]. Regarding pancreatic injury, it is also rare but severe complications occurring in $2-5 \%$ of blunt abdominal trauma $[33,34]$ and are usually associated with injuries of other intra- 
abdominal organs. Isolated pancreatic rupture is quite rare due to its location in a relatively protected retroperitoneal area of the abdominal cavity $[35,36]$. In the acute setting, clinical symptoms are often initially subtle, leading to delay in diagnosis, increase in morbidity and mortality of up to $60 \%$ [37]. The common complications of pancreatic injuries are a pancreatic fistula, intra-abdominal abscess, pancreatitis, pseudocyst and sepsis [38]

The treatment options for an extrahepatic biliary leak have broadened. Until recently, such injuries usually mandated surgical repair utilizing debridement and closure with or without T-tube; patch closure using gallbladder, cystic duct, vein, serosa or jejunum; biliary-enteric anastomosis using duodenum or jejunum; or ligation and drainage with plans for subsequent enteric diversion [39]. For pancreatic injuries, the integrity of the main pancreatic duct (MPD) is the most important determinant of prognosis in these patients, with disruption to the MPD an indication for laparotomy $[40,41]$.

Pancreatic injuries are classified into five grades according to the Pancreas Injury Scale published in 1990 by the American Association for the Surgery of Trauma [42]. Traditionally, patients with injuries to the MPD (grade III, IV, V) require laparotomy. However, a trial of nonoperative treatment may be considered even in more severe injuries such as isolated grade IV injuries if the patient is haemodynamically stable. Endoscopic stenting and drainage is an attractive minimally invasive therapeutic procedure for haemodynamically stable patients, and it may obviate the need for surgery [43-46].

From the aforementioned, we can get an integrated image of the management of the acute extrahepatic biliary system and pancreatic injuries. However, for chronic deformities and altered anatomy, the literature has scanty resources. The presentation of our patient with septic shock and cholangitis necessitated staged approach starting with stabilization, decompression and antimicrobial therapy, with definitive management to prevent recurrence deferred to be done on an elective basis.

According to Tokyo Guidelines 2018 (TG18) [47], our patient met the criteria for the definite diagnosis of acute cholangitis. Moreover, applying the TG18/TG13 severity assessment criteria for acute cholangitis [47,48], our patient classified as having Grade III (severe) acute cholangitis owing to his cardiovascular, hepatic and renal dysfunction sustained during the acute attack.

Over the past decade, the use of surgical and percutaneous drainage has declined. On the contrary decompression via ERCP has risen. In the setting of acute purulent cholangitis, timely performed ERCP is the standard therapy for obtaining source control providing a reliable option with increased diagnostic and therapeutic effectiveness and decreased morbidity and mortality rates [49-52]. Accordingly, urgent decompression was attempted via ERCP. Unfortunately, the changed anatomy where the distal CBD was completely disconnected from the ampulla of Vater, which rendered the cannulation of his CBD impossible even with precut needle knife. PTC was performed as a second line, and despite being beneficial in delineating the abnormal anatomy, it did not drain the biliary system adequately. Upsizing of PTC had been essential with changing towards external/internal drainage at a later stage. Utilization of the full capabilities of the interventional radiology service in our institution altogether with the judicious performance of intensive care plays a crucial role in getting through such a critical clinical course successfully with eventual recuperation and regaining the fitness for definitive management. In addition, MDT approach in various occasions through the management process enabled the surgical team to reach the final stage of definitive surgery safely.
Planning for surgery commenced with verifying the official reports of his old trauma, in addition to CT imaging. The question was how the patient remained silent for 35 years and all of the sudden experienced overwhelming sepsis and cholangitis. Review of the literature to find a reason why the patient had not experienced exocrine pancreatic failure after disruption of his pancreatic main duct with no history of steatorrhea nor weight loss, it was found that, it would be a possibility of a pancreatic divisum, which is a relatively rare congenital anomaly where the main duct remained in the dorsal pancreas draining through the duct of Santorini [52]. In such a case, the main pancreatic duct flows into the duodenum via minor papilla, however, at ERCP the minor papilla was not visualized. Moreover, there were no reports regarding blunt trauma cases in patients with divisum, to our knowledge.

After optimization of the general condition through cardiology, dietitian, endocrinology and anaesthesia review the patient was admitted for definitive surgery.

\section{Operative details}

Midline incision with $\mathrm{J}$ extension to the right side from inferior aspect was performed where extensive adhesions were encountered from previous surgeries. Careful adhesiolysis was done to separate the liver from the colon, small bowel and stomach. Prior cholecystojejunostomy was identified and dismantled with Endo GIA stapler and the gallbladder was dissected down to the confluence with the CBD. Then the CBD and the CHD were clearly identified. The CBD was transected distally, and its stump was closed in a continuous fashion after ensuring that, no stones were left in the remnant. The CHD was then divided with the removal of the gallbladder and the CBD. The jejunum was divided with endo GIA at the same site as the previous cheloecystojejunostomy. Jejunojejunal anastomosis was created with the alimentary limb being anastomosed to the biliary limb with endo GIA and overrunning sutures. Finally, hepaticojejunostomy was created with Maxon 5 - 0 sutures in an interrupted fashion. The mesenteric defect was then closed to prevent a possible internal herniation. No bile leak was found post anastomosis. The peritoneal cavity was thoroughly washed with warm saline. Two $15 \mathrm{Fr}$ JP drains were inserted, one at the site of biliary anastomosis and the other in the peritoneal cavity. Wound infusion catheters were placed for postoperative analgesia using bupivacaine. Hemostasis was achieved, and the abdominal wall was closed in 2 layers with looped No 1 PDS, while the skin was closed with staples.

The patient went through an uneventful smooth postoperative course with no single complication and came for follow up after discharge to our clinic, symptom-free, where skin clips were removed, and the patient reassured that his surgery was successful.

\section{Conclusions}

- Definitive surgery remained the mainstay of treatment for extrahepatic biliary and pancreatic trauma to prevent future lifethreatening complications.

- MDT approach is the optimal choice for dealing with critically ill patients.

- Utilization of the full capabilities of endoscopic and interventional radiology services deemed mandatory for urgent and timely management.

\section{References}

1. Boey JH, Way LW (1980) Acute cholangitis. Ann Surg 191: 264

2. Kimura Y, Takada T, Kawarada Y (2007) Definitions, pathophysiology, and 
Citation: Alsaati H, Omar I, Sharif O, Alhamid WA (2018) Management of Acute Ascending Cholangitis with Septic Shock on Top of Altered Anatomy after Old Road Traffic Accident: A Case Report. J Clin Case Rep 8: 1177. doi: 10.4172/2165-7920.10001177

Page 6 of 6

epidemiology of acute cholangitis and cholecystitis: Tokyo guidelines. J Hepatobiliary Pancreat Surg 14: 15

3. Huibregtse K, Carr-Locke DL, Cremer M (1992) Biliary stent occlusion: A problem solved with self-expanding metal stents? European Wallstent Study Group. Endoscopy 24: 391.

4. Zeuge U, Fehr M, Meyenberger C, Sulz MC (2014) Mind the Sump! - Diagnostic challenge of a rare complication of choledochoduodenostomy. Case Rep Gastroenterol 8: 358-363.

5. Leppard WM, Shary TM, Adams DB, Morgan KA (2011) Choledochoduodenostomy: Is it really so bad? J Gastrointest Surg 15: 754757.

6. Reynolds BM, Dargan EL (1959) Acute obstructive cholangitis: A distinc clinical syndrome. Ann Surg 150: 299-303.

7. Sung JY, Costerton JW, Shaffer EA (1992) Defense system in the biliary tract against bacterial infection. Digest Dis Sci 37: 689-696.

8. Andrew DJ, Johnson SE (1970) Acute suppurative cholangitis, a medical and surgical emergency: A review of ten years' experience emphasizing early recognition. Am J Gastroenterol 54: 141-154.

9. Shimada H, Nakagawara G, Kobayashi M (1984) Pathogenesis and clinica features of acute cholangitis accompanied by shock. Japanese J Surg 14: 269277.

10. Bakkum, Loffeld (2017) Ascending cholangitis due to heavy lifting. Case Rep Gastroenterol 11: 500-503.

11. Touzios JG, Krzywda B, Nakeeb A, Pitt HA (2005) Exercise-induced cholangitis and pancreatitis. HPB (Oxford) 7: 124-128.

12. Bong-Koo K, Park SM, Byung-Wook K, Kim JS, Kim JH, et al. (2015) Cholangitis secondary to food material impaction in the common bile duct through a choledochoduodenal fistula. Clin Endosc 48: 265-267.

13. Croghan, Stefanie M (2018) Ascending cholangitis: Rare presentation of a ruptured right-sided renal angiomyolipoma. J Surg Case Rep p: 10.

14. Pattarapuntakul T, Ovartlarnporn B, Sottisuporn J (2018) Mucinous cystic neoplasm of the liver with extrahepatic growth presenting with ascending cholangitis diagnosed by endoscopic ultrasound features: A case report. J Med Case Rep 12: 33.

15. Scaife M, Abegglen R, Vila C, Stahlfeld K (2018) Abnormal presentation of ascending cholangitis. Clin Case Rep 6: 1172-1173.

16. Rosh AJ, Manko JA, Santen S (2014) Cholangitis in emergency medicine. Brenner//Medscape 2012

17. Leung JWC, Ling TKW, Chan RCY (1994) Antibiotics, biliary sepsis, and bile duct stones. Gastrointestinal Endoscopy 40: 716-721.

18. Sinanan MN (1992) Acute cholangitis. Infect Dis Clin Am 6: 571-599.

19. Yang MH, Chen TH, Wang SE (2008) Biochemical predictors for absence of common bile duct stones in patients undergoing laparoscopic cholecystectomy. Surg Endosc 22: 1620-1624.

20. Cheon YK, Lehman GA (2006) Identification of risk factors for stone recurrence after endoscopic treatment of bile duct stones. Eur J Gastroenterol Hepato 18: $461-464$

21. Keizman D, Shalom MI, Konikoff FM (2006) Recurrent symptomatic common bile duct stones after endoscopic stone extraction in elderly patients. Gastrointest Endosc 64: 60-65.

22. Lewis HI, Sen A, Cockerell CO (2011) Difficulty in walking 10 years after gastric surgery. Eur J Gastroenterol Hepatol 23: 952-953.

23. Harrison E, Stokes W, Martin JE (2013) Frontline. Gastroenterol 4: 282-287.

24. Gabrielli M, Bonazzi P, Scarpellini E (2011) Prevalence of small intestina bacterial overgrowth in Parkinson's disease. Mov Disord 26: 889-892.

25. Marie I, Ducrotté $P$, Denis $P$ (2009) Small intestinal bacterial overgrowth in systemic sclerosis. Rheumatol 48: 1314-1319.

26. Ramia JM, Gutierrez G, Garrote D, Mansilla A, Villar J, et al. (2005) Isolated extrahepatic bile duct rupture in blunt abdominal trauma. Am J Emerg Med 23 231-232.

27. Simstein N (2000) Isolated blunt trauma injury to the hepatic duct. Int Surg 85 $55-56$.
28. Sanford Z, Abdolmaali K, Robinson D, Denning D (2015) Blunt trauma: An uncommon cause of common bile duct injury. Trauma Case Rep 1: 44-48.

29. Feliciano DV (1994) Biliary injuries as a result of blunt and penetrating trauma. Surg Clin N Am 74: 897-907.

30. Lee D, Zacher J, Vogel TT (1976) Primary repair in transection of duodenum with avulsion of the common duct. Arch Surg 111: 592-593.

31. Fletcher WS (1972) Non penetrating trauma to the gallbladder and extrahepatic bile ducts. Surg Clin North Am 52: 711-717.

32. Maier WP, Lightfoot WP, Rosemond GP (1968) Extrahepatic biliary ducta injury in closed trauma. Am J Surg 116: 103-108.

33. Lin BC, Chen RJ, Fang JF, Hsu YP, Kao YC, et al. (2004) Management of blunt major pancreatic injury. J Trauma 56: 774-778.

34. Subramanian A, Dente CJ, Feliciano DV (2007) The management of pancreatic trauma in the modern era. Surg Clin N Am 87: 1515-1532.

35. Levine RA, Bank MA (2011) Traumatic transection of the pancreas: Case of delayed presentation. JOP 12: 47-49.

36. Vezakis A, Koutoulidis V, Fragulidis G, Polymeneas G, Polydorou A (2014) Complete traumatic main pancreatic duct disruption treated endoscopically: A case report. J Med Case Rep 8: 173.

37. Krishna A, Kaul PB, Murali MV (1992) Isolated extrahepatic bile duct injury: Diagnosis and surgical management. Pediatr Surg Int 7: 143-145.

38. Kshirsagar AY, Vekariya MA, Pednekar AS, Mahna A, Patankar R, et al. (2015) Isolated pancreatic tail injury: A rare presentation. Ann Med Surg 4: 230-232.

39. Bade PG, Thomson SR (1989) Surgical options in traumatic injury to the extrahepatic biliary tract. Br J Surg 76: 256-258.

40. Degiannis E, Glapa M, Loukogeorgakis SP, Smith MD (2008) Management of pancreatic trauma. Injury 39: 21-29.

41. Bhasin DK, Surinder SR, Rawal P (2009) Endoscopic retrograde pancreatography in pancreatic trauma: Need to break the mental barrier. $J$ Gastroenterol Hepatol 24: 720-728.

42. Moore EE, Cogbill TH, Malangoni MA, Jurkovich GJ, Champion HR, et al. (1990) Organ injury scaling, II: Pancreas, duodenum, small bowel, colon and rectum. J Trauma 30: 1427-1429.

43. Hiremath B, Hegde N (2014) Non-operative management of a grade IV pancreatic injury. BMJ Case Rep 2: 1.

44. Kottapalli, Chakravarty D (2018) Complete pancreatic duct disruption in an isolated pancreatic injury: Successful endoscopic management. Oxford Med Case Rep 3: 44-46.

45. Kiriyama S, Kozaka K, Takada T, Strasberg SM, Pitt HA, et al. (2018) Tokyo Guidelines 2018: diagnostic criteria and severity grading of acute cholangitis. $J$ Hepatobiliary Pancreat Sci 25: 17-30.

46. Kiriyama S, Takada T, Strasberg SM, Solomkin JS, Mayumi T, et al. (2012) New diagnostic criteria and severity assessment of acute cholangitis in revised Tokyo Guidelines. J Hepatobiliary Pancreat Sci 19: 548-556.

47. McNabb-Baltar J, Trinh QD, Barkun AN (2013) Biliary drainage method and temporal trends in patients admitted with cholangitis: A national audit. Can $J$ Gastroenterol 27: 513-518.

48. Buyukasik K, Toros AB, Bektas H, Ari A, Deniz MM (2013) "Diagnostic and Therapeutic Value of ERCP in Acute Cholangitis," ISRN Gastroenterol 2013: 4.

49. Mok SR, Mannino CL, Malin J, Drew ME, Henry P, et al. (2012) Does the urgency of endoscopic retrograde cholangiopancreatography (ercp)/percutaneous biliary drainage ( $\mathrm{pbd}$ ) impact mortality and disease related complications in ascending cholangitis? (deim-i study). J Interv Gastroenterol 2: 161-167.

50. Miura F, Takada T, Kawarada Y, Nimura Y, Wada K, et al. (2007) Flowcharts for the diagnosis and treatment of acute cholangitis and cholecystitis: Tokyo guidelines. J Hepatobiliary Pancreat Surg 14: 27-34.

51. Jamal MM, Yamini D, Singson Z (2011) Decreasing hospitalization and inhospital mortality related to cholangitis in the United States. J Clin Gastroentero 45: 92-96

52. Kozu T, Suda K, Toki F (1995) Pancreatic development and anatomical variation. Gastrointest Endosc Clin N Am 5: 1-30. 